\title{
Physical inactivity associated with the risk of non-communicable diseases in Japanese working mothers with young children: A cross-sectional study in Nagano city, Japan
}

\author{
YOSHIO SUZUKI ${ }^{1,2}$, KEISHOKU SAKURABA ${ }^{1,2}$, TOKIKO SHINJO ${ }^{1}$, \\ ASAKO MARUYAMA-NAGAO ${ }^{2}$, ATSUKO NAKANIIDA ${ }^{2}$, HARUKA KADOYA ${ }^{2}$, \\ MARIKA SHIBATA ${ }^{2}$, TAKEHISA MATSUKAWA ${ }^{3}$, HIROAKI ITOH ${ }^{3}$ and KAZUHITO YOKOYAMA ${ }^{3}$ \\ ${ }^{1}$ Graduate School of Health and Sports Science; ${ }^{2}$ Faculty of Health and Sports Science, \\ Juntendo University, Chiba 270-1695; ${ }^{3}$ Department of Epidemiology and Environmental Health, \\ Juntendo University Faculty of Medicine, Tokyo 113-0033, Japan
}

Received December 26, 2016; Accepted February 17, 2017

DOI: $10.3892 / \mathrm{etm} .2017 .4311$

\begin{abstract}
Physical activity helps to prevent the development of chronic non-communicable diseases. However, childbearing generally reduces parents' level of physical activity, particularly in mothers. Therefore, mothers with young children generally have lower levels of physical activity and have a higher risk of developing non-communicable diseases. The aim of the present study was to examine this risk in Japanese working mothers with young children. A cross-sectional study was conducted in four nursery schools in Nagano city, Japan. All mothers were asked to complete a questionnaire regarding abnormal findings at their proximate annual medical examination, and were asked to record their normal physical activity. A total of 182 mothers completed the questionnaires, and 36 reported having abnormal findings (ABN group). Mothers in the ABN group were significantly older than those without abnormal findings (NOR; $\mathrm{P}=0.043$ ). No significant differences in physical activity were observed between the two groups; however, mothers in the $\mathrm{ABN}$ group spent a significantly longer time sitting than those in the NOR group $(\mathrm{P}=0.028)$. Regarding socioeconomic characteristics, mothers in the ABN group had a significantly higher educational background $(\mathrm{P}=0.040)$ and a higher annual family income $(\mathrm{P}<0.001)$ compared with those in the NOR group, and significantly more mothers held full-time jobs (55.9 vs. $36.0 \%$; $\mathrm{P}=0.005$ ). Full-time working mothers typically had a significantly higher family income $(\mathrm{P}<0.001)$ and spent a significantly longer time sitting $(\mathrm{P}<0.001)$ compared with mothers in part-time and
\end{abstract}

Correspondence to: Dr Yoshio Suzuki, Graduate School of Health and Sports Science, Juntendo University, 1-1 Hiragagakuendai, Inzai, Chiba 270-1695, Japan

E-mail: yssuzuki@juntendo.ac.jp

Key words: physical inactivity, sedentary, non-communicable disease, occupation, mother, sitting other work. Therefore, the results of the present study suggest that sedentary lifestyles, namely the amount of time spent sitting, may increase the risk of Japanese working mothers with young children developing non-communicable diseases.

\section{Introduction}

It is well known that physical inactivity increases the risk of developing chronic non-communicable diseases, including coronary heart disease, type 2 diabetes, breast and colon cancers, and shortens life expectancy (1), whereas regular physical activity helps to prevent the occurrence of diseases such as cardiovascular disease, diabetes, cancer, hypertension, obesity, depression, osteoporosis and premature death (2).

Previous studies have demonstrated that childbearing reduces physical activity levels among parents $(3,4)$, particularly in mothers during pregnancy and for $\sim 6$ months following birth (5). Parenthood is associated with more sedentary lifestyles in both fathers and mothers $(4,6)$, and typically mothers were less active than fathers (4).

In Japan, it has been reported that employed women have significantly lower physical activity levels than unemployed women (7). Traditionally, Japanese mothers have larger roles in childcare than fathers. Among Japanese mothers with children 0-6 years old in nursery school, $<20 \%$ reported exercising or attempting to exercise regularly (8). Furthermore, rural areas tend to lack public transportation such as buses and trains, and working mothers with young children in these regions typically depend on individual car transportation. This may reduce the opportunities for mothers to walk, stand and hold or lift their child or baggage and reduce the physical activity.

The physical inactivity in working mothers in rural areas may increase the risk of developing non-communicable diseases, and the lifestyle factors that affect the risk remain to be elucidated. In Japan all citizens are required to take an annual medical examination, where their risk of non-communicable diseases is assessed and the results are fed back to the individuals. The present study hypothesized that physical activity/inactivity affected the risk of the non-communicable 
diseases that may be identified during the annual medical examinations of working mothers in rural areas with young children. The aim of the present study was to investigate how medical examination results correlate with physical activity levels and the socioeconomic backgrounds of working mothers with young children in rural cities. The current study describes a cross-sectional study conducted in Nagano city, a typical Japanese rural city.

\section{Materials and methods}

Study area. A cross-sectional study was conducted in Nagano city, Japan. Nagano city is the prefectural capital of Nagano Prefecture, which is located in the geographic center of Honshu, the main island of Japan. The city is situated in a mountainous area 80 min away from Tokyo by express train. Japanese culture is generally divided into eastern Japanese culture, represented by Tokyo, and western Japanese culture represented by Osaka and Kyoto. As Nagano is located in the middle of Honshu, its culture has features in both eastern and western aspects. Nagano city is one of 48 core cities, which have a population between 200 and 500 thousand individuals. Therefore, the present study selected Nagano city assuming people in Nagano city represent an average Japanese lifestyle in this size of cities in rural areas.

In 2010, Nagano's gross prefectural production placed it 16 th out of the 47 prefectures in Japan (9). The majority of Nagano residents are employed in the service industry and a further $9.8 \%$ are employed in the agriculture, forestry and fisheries sectors (10). Due to the mountainous terrain, the public transportation system is limited, and $74.9 \%$ of households own $>1$ automobiles, which is the highest percentage among all prefectures in Japan (mean, 40.7\%) (11).

In 2010, the population of Nagano city was 381,511 and there were 90,755 households, which contained married couples, among which there were 32,562 working mothers (12). The top three industries in which working mothers were employed in were the clerical, service, and professional/technical industries, in decreasing order (12). The mean annual household income in Nagano was 302.6 million yen, 379th among all 1,741 municipalities in Japan (13).

Participants. The present study was conducted as part of a survey to investigate childhood health and physical activity in Nagano (14). In November 2012, mothers of 645 children attending four nursery schools that were part of the Suginoko Group, the largest private nursery school foundation in Nagano, gave written informed consent to participate in the study. They were provided with an explanation of the study, consent form and questionnaire at the nursery schools, and consent forms and questionnaires were recovered within a month. Subjects who provided incomplete responses to the questions on physical activity and medical examination results were excluded, and the remaining 182 (28.2\%) mothers were enrolled in the present study.

The present study was conducted according to the guidelines laid down in the Declaration of Helsinki. All procedures involving human subjects were approved by the Ethics Committee of Juntendo University School of Medicine (\#2012057).
Study variables. The data provided by each subject in the questionnaire was as follows: Age, body weight, height, drinking habits, smoking habits, results of latest medical examination (if they had abnormal findings in results, yes or no), educational background, type of employment, occupation, whether they worked night shifts (yes or no), annual family income and current exercise activity level. The highest academic background achieved categorized the educational background of individuals: Junior high school, high school, junior college/vocational school, university and graduate school. Employment type was selected from part-time, full-time and other. Options for occupation were as follows: Professional/technical, administrative, clerical, sales, service, agriculture, forestry, fishery, transport/communication, production/construction and others.

In Japan, all citizens are required to undergo an annual medical examinations. The examination records the following (reference ranges are provided in brackets): Body weight, height, body mass index (BMI; $<25 \mathrm{~kg} / \mathrm{m}^{2}$ ), waist circumference $(<90 \mathrm{~cm}$ ), blood pressure (systolic $<130 \mathrm{mmHg}$; diastolic $<85 \mathrm{mmHg}$ ). Blood tests are also performed to measure levels of hemoglobin $(>12.0 \mathrm{~g} / \mathrm{dl})$, triglycerides $(<150 \mathrm{mg} / \mathrm{dl})$, high-density lipoprotein cholesterol (>39 mg/dl), low-density lipoprotein cholesterol $(<120 \mathrm{mg} / \mathrm{dl})$, aspartate transaminase $(<31 \mathrm{U} / \mathrm{l})$, alanine transaminase $(<31 \mathrm{U} / \mathrm{l})$, gamma-glutamyl transferase $(<51 \mathrm{U} / 1)$ and hemoglobin A1c $(<5.6 \%$ as determined by the National Glycohemoglobin Standardization Program) (15). If any of the aforementioned variables were outside of the reference ranges, mothers were instructed to answer 'yes' to abnormal findings (and were considered the ABN group, while individuals with no abnormal findings were considered the NOR group). These reference ranges were set to screen the risk of non-communicable diseases, and individuals with abnormal finding are instructed to consult a doctor or receive public health guidance. Current exercise activity levels were assessed using the Japanese version of the International Physical Activity Questionnaire-Short Form (IPAQ-SF) $(16,17)$.

Statistical analysis. The socioeconomic backgrounds and physical activity levels of mothers who reported abnormal findings at their proximal medical examination were compared with those who did not report abnormal findings. The average annual income of ABN and NOR were calculated assuming the income was a mean of the option range; e.g., 0.5 and 1.5 for the options ' $<1$ ' and '1-2', respectively. The Mann-Whitney U test or Kruskal-Wallis test was used to analyze the mean difference in continuous variables, and the $\chi^{2}$ test with or without linear-by-linear association was used to analyze differences in the proportion of categorical data when multiple categories were ordered.

To assess the risk of background characteristics on health, binary logistic regression was performed using medical abnormal finding $(\mathrm{NO}=1$ and $\mathrm{YES}=2)$ as a response variable, and age and background characteristics as covariates. In logistic analysis, educational background was analyzed as numerical data, and annual family income was coded so that an increase of 1 corresponded to an increase of 1,000,000 yen.

Missing data were excluded from the analysis in a pairwise manner. All statistical analyses were performed using 
Table I. Participants' background characteristics.

\begin{tabular}{lccc}
\hline Parameter & ABN n=36 & NOR n=146 & P-value \\
\hline Age, y & $37.4 \pm 5.0$ & $36.0 \pm 4.7$ & $0.043^{\mathrm{a}}$ \\
Height, cm & $158.2 \pm 5.6$ & $158.5 \pm 6.5$ & 0.627 \\
Weight, kg & $52.5 \pm 9.4$ & $51.2 \pm 6.3$ & 0.822 \\
BMI, kg $/ \mathrm{m}^{2}$ & $21.0 \pm 3.6$ & $20.4 \pm 3.0$ & 0.773 \\
Drinking, \% & 52.8 & 52.7 & 1.000 \\
Smoking, \% & 5.6 & 8.9 & 0.739
\end{tabular}

Data are expressed as the mean \pm standard deviation or percentage. ${ }^{\mathrm{a}} \mathrm{P}<0.05$. ABN, abnormal group; NOR, normal group; BMI, body mass index.

SPSS software version 19 (IBM SPSS, Armonk, NY, USA). $\mathrm{P}<0.05$ was considered to indicate a statistically significant difference.

\section{Results}

Physical characteristics of participants. Among the 182 participants, $36(19.8 \%)$ reported having abnormal findings at their latest annual medical examination. Participants who had abnormal findings were classified into the ABN group ( $n=36)$, and the rest $(n=146)$ were classified into the NOR group. The mean age of mothers in the ABN group (37.4 years) was significantly higher than that of the NOR group (36.0 years; $\mathrm{P}=0.043)$. No significant differences between the groups were observed in any anthropometric parameters (height, weight or BMI) or in drinking and smoking habits (Table I).

Educational and socioeconomic background. The educational and socioeconomic backgrounds of participants are presented in Table II. Of all participants, $\sim 50 \%$ had graduated from junior college or vocational school, $\sim 30 \%$ were high school graduates and $\sim 20 \%$ were university graduates. All mothers in the ABN group had at least graduated from high school and $25 \%$ had graduated from university or graduate school. In the NOR group, by contrast, $1.4 \%$ of participants were not high school graduates and only $13.8 \%$ had graduated from university or graduate school. Mothers in the ABN group therefore had significantly higher educational backgrounds than those in the NOR group $(\mathrm{P}=0.040)$.

Significant differences were also observed in employment type $(\mathrm{P}=0.005)$. In the $\mathrm{ABN}$ group, $>50 \%$ of mothers worked full-time; whereas only $36.0 \%$ of the mothers worked full-time in the NOR group. Mothers in the ABN group worked primarily in the clerical (42.9\%) and professional/technical $(31.4 \%)$ fields, whereas the employment types of those in the NOR group were more varied. However, differences in employment type were not statistically significant (Table II).

Annual family income had a bimodal distribution, with peaks at 7-8 million yen and 8-10 million yen for the ABN group, and a unimodal, bell-shaped distribution with a significantly lower peak at 4-5 million yen, for the NOR group; the distributions were significantly different $(\mathrm{P}<0.001$; Table II). In addition, the mean annual family income of the ABN group
Table II. Participants' socioeconomic characteristics.

\begin{tabular}{|c|c|c|c|}
\hline Parameter & $\mathrm{ABN}(\%)$ & NOR $(\%)$ & P-value \\
\hline Education, $\mathrm{n}$ & 36 & 145 & \\
\hline Junior high school & 0.0 & 1.4 & $0.040^{\mathrm{a}}$ \\
\hline High school & 27.8 & 29.0 & \\
\hline $\begin{array}{l}\text { Junior college/ } \\
\text { vocational school }\end{array}$ & 47.2 & 55.9 & \\
\hline University & 19.4 & 13.1 & \\
\hline Graduate school & 5.6 & 0.7 & \\
\hline Employment type, $\mathrm{n}$ & 34 & 139 & \\
\hline Part-time & 41.2 & 43.2 & $0.005^{\mathrm{a}}$ \\
\hline Full-time & 55.9 & 36.0 & \\
\hline Other & 2.9 & 20.9 & \\
\hline Occupation, $\mathrm{n}$ & 35 & 145 & \\
\hline Professional/technical & 31.4 & 20.0 & 0.054 \\
\hline Administrative & 0.0 & 0.7 & \\
\hline Clerical & 42.9 & 19.3 & \\
\hline Sales & 2.9 & 4.8 & \\
\hline Service & 8.6 & 13.1 & \\
\hline $\begin{array}{l}\text { Agriculture, forestry, } \\
\text { and fishery }\end{array}$ & 0.0 & 1.4 & \\
\hline Transport/communication & 0.0 & 1.4 & \\
\hline Production/construction & 2.9 & 6.9 & \\
\hline Others & 11.4 & 32.4 & \\
\hline $\begin{array}{l}\text { Family income, } \mathrm{n} \\
\text { (million yen) }\end{array}$ & 33 & 134 & \\
\hline$<1$ & 0.0 & 6.0 & $<0.001^{\mathrm{a}}$ \\
\hline $1-2$ & 0.0 & 4.5 & \\
\hline $2-3$ & 9.1 & 11.2 & \\
\hline $3-4$ & 3.0 & 15.7 & \\
\hline $4-5$ & 15.2 & 17.9 & \\
\hline $5-6$ & 9.1 & 15.7 & \\
\hline $6-7$ & 27.3 & 13.4 & \\
\hline $7-8$ & 9.1 & 8.2 & \\
\hline $8-10$ & 27.30 & 7.5 & \\
\hline
\end{tabular}

Number of the total answers (n) was $<182$ in certain cases because some participants did not answer all questions in this questionnaire. All data are presented as percentages, unless otherwise specified. ${ }^{\mathrm{a}} \mathrm{P}<0.05$. ABN, abnormal group; NOR, normal group.

(6.4 million yen) was significantly higher than that of NOR group (4.8 million yen; $\mathrm{P}<0.001$; Table II).

Physical activity. Participants' physical activity was assessed using the Japanese version of the IPAQ-SF $(16,17)$ (Table III). Neither group scored within the median percentile for vigorous or moderate physical activity. In the $\mathrm{ABN}$ group, $>50 \%$ answered that they spent no time walking with the intention of conducting physical activity and median walking activity was markedly lower in the ABN group compared with the NOR group. Similarly, median total physical activity was markedly lower in the ABN group compared with the NOR group. However, the only significant difference in physical 
Table III. Participants' physical activity assessed using the short form Japanese version of the international physical activity questionnaire.

\begin{tabular}{|c|c|c|c|}
\hline Parameter & $\mathrm{ABN}, \mathrm{n}=36$ & NOR, n=146 & P-value \\
\hline $\begin{array}{l}\text { Vigorous PA, } \\
\text { MET·min/week }\end{array}$ & $46.7 \pm 179.9$ & $40.5 \pm 160.6$ & 0.956 \\
\hline $25 \%$ & 0.0 & 0.0 & \\
\hline Median & 0.0 & 0.0 & \\
\hline $75 \%$ & 0.0 & 0.0 & \\
\hline $\begin{array}{l}\text { Moderate PA, } \\
\text { MET·min/week }\end{array}$ & $133.0 \pm 368.4$ & $72.1 \pm 201.8$ & 0.224 \\
\hline $25 \%$ & 0.0 & 0.0 & \\
\hline Median & 0.0 & 0.0 & \\
\hline $75 \%$ & 29.8 & 47.3 & \\
\hline $\begin{array}{l}\text { Walking, MET. } \\
\text { min/week }\end{array}$ & $101.9 \pm 209.6$ & $139.4 \pm 266.5$ & 0.094 \\
\hline $25 \%$ & 0.0 & 28.0 & \\
\hline Median & 44.8 & 141.0 & \\
\hline $75 \%$ & 424.0 & 449.5 & \\
\hline $\begin{array}{l}\text { Total PA, } \\
\text { MET·min/week }\end{array}$ & $281.6 \pm 577.3$ & $251.9 \pm 402.7$ & 0.078 \\
\hline $25 \%$ & 0.0 & 16.3 & \\
\hline Median & 31.0 & 71.0 & \\
\hline $75 \%$ & 194.0 & 287.3 & \\
\hline $\begin{array}{l}\text { Time spent } \\
\text { sitting, min/day }\end{array}$ & $332 \pm 195$ & $266 \pm 228$ & $0.026^{\mathrm{a}}$ \\
\hline $25 \%$ & 180 & 90 & \\
\hline Median & 300 & 180 & \\
\hline $75 \%$ & 480 & 375 & \\
\hline
\end{tabular}

Data are expressed as mean \pm standard deviation and 25,50 (median), and 75 percentiles. Mean difference was assessed by Mann-Whitney $\mathrm{U}$ test. ${ }^{\mathrm{a}} \mathrm{P}<0.05$. $\mathrm{ABN}$, abnormal group; NOR, normal group; $\mathrm{PA}$, physical activity; MET, Metabolic Equivalent of Task.

activity between groups was in time spent sitting. Mothers in the NOR group spent significantly less time sitting than those in the $\mathrm{ABN}$ group $(\mathrm{P}=0.026$; Table III $)$.

Socioeconomic background and health. The only significant difference in socioeconomic parameters between the ABN and NOR groups was in annual family income; mothers in the ABN group were found to have significantly higher annual family incomes than those in the NOR group $(\mathrm{P}=0.001)$ when controlling for age. Every 1,000,000 yen increase in income increased the likelihood of abnormal medical findings by 1.4 times (Table IV).

Binary logistic regression was performed using abnormal range $(1=\mathrm{NO}, 2=\mathrm{YES})$ at proximal medical examination as the responsible parameter, and age and background characteristics as covariates.

Mothers' working style was demonstrated to be associated with annual family income. Full time working mothers had a significantly higher family income compared with part time or
Table IV. The medical examination abnormal findings and background characteristics controlled for age.

\begin{tabular}{lllll}
\hline & & \multicolumn{2}{c}{$95 \%$ CI limits } & \\
\cline { 3 - 4 } Background & OR & Lower & Upper & P-value \\
\hline Drinking & 0.997 & 0.477 & 2.083 & 0.994 \\
Smoking $^{\mathrm{a}}$ & 1.464 & 0.310 & 6.910 & 0.630 \\
Education $^{\mathrm{b}}$ & 1.408 & 0.847 & 2.340 & 0.187 \\
Night shift $^{\mathrm{a}}$ & 0.665 & 0.169 & 2.620 & 0.560 \\
Family income $^{\mathrm{c}}$ & 1.407 & 1.156 & 1.713 & $0.001^{\mathrm{d}}$ \\
& & & &
\end{tabular}

${ }^{\mathrm{a}} \mathrm{YES}=1, \mathrm{NO}=2$; 'increase of 1 corresponds to one step higher educational level; cincrease of 1 corresponds to an increase of one million yen in annual family income ${ }^{\mathrm{d}} \mathrm{P}<0.05$. CI, confidence interval; OR, odds ratio.

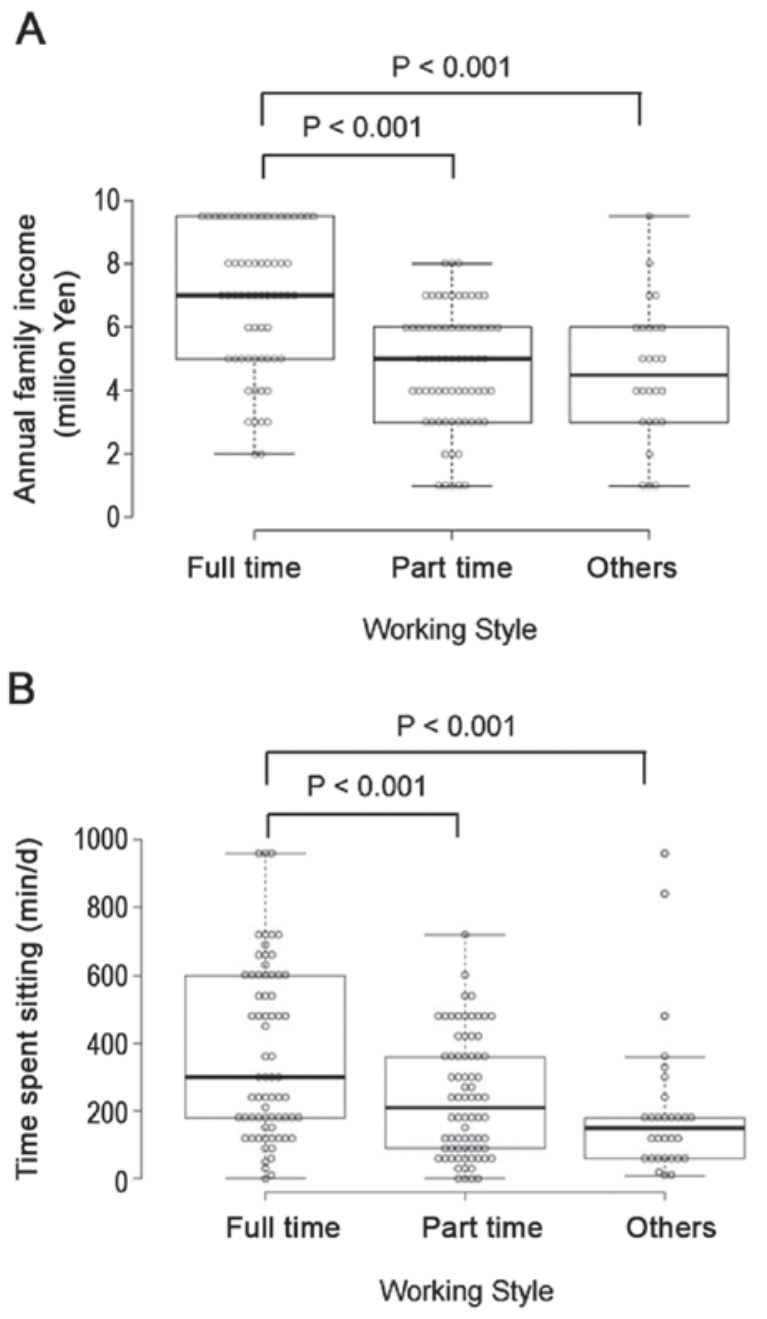

Figure 1. Effects of working style on family income and physical activity levels. (A) Annual family income was assumed to be the mean of the ranges indicated in the chosen alternative. (B) Mean difference in time spent sitting was analyzed using the Kruskal-Wallis test followed by pairwise comparison.

other working mothers $(\mathrm{P}<0.001$; Fig. $1 \mathrm{~A})$. Mothers' working style was also associated with the amount of time spent sitting. Full time working mothers spent significantly longer sitting 
compared with part time $(\mathrm{P}<0.001)$ or other working mothers $(\mathrm{P}<0.001$; Fig. 1B).

\section{Discussion}

In the present study, the health condition of mothers with children at nursery school in Nagano, Japan, was investigated. Of the participants, $19.8 \%$ reported abnormal findings at their most recent medical examination. The mean age was higher in the $\mathrm{ABN}$ group, however no differences were observed in smoking or drinking habits between groups. The ABN group had a significantly higher educational background and annual family income, and a significantly higher percentage of participants had full-time jobs than the NOR group. No significant differences were observed in physical activity levels between the two groups; however, the ABN group spent significantly more time sitting than the NOR group. When data were controlled for age, only family income was significantly associated with abnormal medical findings; every one million yen increase in annual income increased the likelihood of abnormal findings by 1.4 times.

In the present study, it was hypothesized that physical activities affected the risk of the non-communicable diseases that may be identified in annual medical examinations. However, no significant difference was observed in physical activities assessed by IPAQ-SF. Despite the reliability and validity of IPAQ-SF previously being confirmed (17), a recent systematic review determined that overall physical activity as assessed by the IPAQ-SF had a negligible to small correlation with physical activity observed by objective measuring devices (18). Therefore, the absence of association between physical activities and abnormal findings in medical examination may be due to the problems with assessing the physical activities.

By contrast, it has been demonstrated that the Japanese version of IPAQ-SF provides highly repeatable data for sitting times (17). Furthermore, although the IPAQ-SF was designed to collect data on physical activity by recording the duration of walking and vigorous and moderate exercise $(16,18)$, intentional exercise activities comprise a small part of total energy expenditure (19). Hamilton et al (19) proposed that the association between sedentary behaviors, particularly sitting, and non-communicable disease and mortality rates was important. The results of the present study demonstrated that mothers in the ABN group spent more time sitting, suggesting an association between physical inactivity and abnormal medical findings. The association between inactivity and the risk of developing non-communicable diseases is in accordance with previous reports identifying the association between inactivity and obesity (20-22).

Regarding the participants' background characteristics, every one million yen increase in annual family income made a participant 1.4 times more likely to have abnormal findings at medical examinations, contrary to results from previous studies. In the USA, it was demonstrated that individuals with lower annual incomes had a higher risk of mortality (23). In Finland, individuals with lower annual incomes had lower self-rated health statuses and a higher prevalence of long-standing illnesses (24). Even in Japan, higher household incomes were reported to be associated with better eating habits, such as consumption of more vegetables and more frequent conversations with friends or family during meals (25).

The discrepancies between the results of the present study and previously published studies may be accounted for by the decrease in physical activity associated with higher family incomes. The majority of mothers in the ABN group held full-time jobs in the clerical, service, and professional/technical industries; therefore, full-time working mothers had a higher family income but also spent more time sitting compared with mothers in part-time and other employment. The discrepancies may therefore be explained, in part, by occupational disparities.

The present study had several limitations. As this was a questionnaire survey, physical activity and health status may have been inaccurately reported. The small sample size may also have caused the results to be misrepresentative, thus the results cannot be fully generalized. Additionally, the marital status and number of children of the participants were not recorded; these factors may influence the opportunity for exercise. The use of active transportation or car use should have been recorded, as this may be associated with physical activity and socioeconomic status. Therefore, future studies should include more parameters to establish whether other factors also have a significant effect on the results.

In conclusion, the results of the present study suggest that sedentary activity may increase the risk of developing non-communicable diseases in Japanese working mothers with young children.

\section{Acknowledgements}

The authors of the present study would like to thank Mr. Yoshiyuki Tanaka, Mr. Kaoru Imai, the staff of the Suginoko Nursery School Group, and the study participants. The present study was supported by the Ministry of Education, Culture, Sports, Science and Technology (MEXT)-Supported Program for the Strategic Research Foundation at Private Universities (grant no. S1101008, Tokyo, Japan).

\section{References}

1. Lee IM, Shiroma EJ, Lobelo F, Puska P, Blair SN and Katzmarzyk PT; Lancet Physical Activity Series Working Group: Effect of physical inactivity on major non-communicable diseases worldwide: An analysis of burden of disease and life expectancy. Lancet 380: 219-229, 2012.

2. Warburton DE, Nicol CW and Bredin SS: Health benefits of physical activity: The evidence. CMAJ 174: 801-809, 2006.

3. Brown WJ and Trost SG: Life transitions and changing physical activity patterns in young women. Am J Prev Med 25: 140-143, 2003.

4. Bellows-Riecken KH and Rhodes RE: A birth of inactivity? A review of physical activity and parenthood. Prev Med 46: 99-110, 2008.

5. Pereira MA, Rifas-Shiman SL, Kleinman KP, Rich-Edwards JW Peterson KE and Gillman MW: Predictors of change in physical activity during and after pregnancy: Project viva. Am J Prev Med 32: 312-319, 2007.

6. Berge JM, Larson N, Bauer KW and Neumark-Sztainer D: Are parents of young children practicing healthy nutrition and physical activity behaviors? Pediatrics 127: 881-887, 2011.

7. Matsushita M, Harada K and Arao T: Socioeconomic position and work, travel, and recreation-related physical activity in Japanese adults: A cross-sectional study. BMC Public Health 15: 916, 2015 
8. Ohtsuki Y, Ishimura Y, Iino N, Hotta K and Aoyagi Y: Health behavior of the child rearing Woman in A City. Iryo Kango Kenkyu 4: 89-94, 2008 (In Japanese).

9. Cabinet Office, Government of Japan: Annual report on prefectural accounts 2001-2013 (Japanese version only). http://www. esri.cao.go.jp/jp/sna/data/data_list/kenmin/files/contents/main h25.html. Accessed February 19, 2017 (In Japanese).

10. Nagano Prefecture: Outlines of population census 2010 (Heisei 22 nen kokuseichosa sangyo-to kihon shukei kekka). http://www3. pref.nagano.lg.jp/tokei/1_kokuchou/H22kakuhou/sangyo/si.pdf. Accessed February 19, 2017 (In Japanese).

11. Ministry of Internal Affairs and Communications of Japan: 2009 National survey of family income and expenditure. Table XVIII. Quantities of major durable goods possessed per 1000 households and percentages of households possessing major durable goods, by area. http://www.e-stat.go.jp/SG1/estat/List. do?bid $=000001027311 \&$ cycode $=0$. Accessed February 19, 2017 (In Japanese).

12. Nagano City: 2010 Population census of Nagano city [Nagano-shi no jinkou-heisei 22 nen kokuseichosa kekka houkokusho] http://www.city.nagano.nagano.jp/uploaded/attachment/99886. pdf. Accessed February 19, 2017 (In Japanese).

13. Ranking of income in Japanese muncipalities 2014 (based on the National survey of family income and expenditure by Ministry of Internal Affairs and Communications of Japan). http://www. nenshuu.net/prefecture/shotoku/past/2014/in_shotoku_city_2014. php. Accessed February 19, 2017 (In Japanese).

14. Yaginuma S, Sakuraba K, Kadoya H, Koibuchi E, Matsukawa T, Ito H, Yokoyama K and Suzuki Y: Early bedtime associated with the salutary breakfast intake in Japanese nursery school. Int Med J 22: 30-32, 2015.

15. Ministry of Health Labour and Welfare of Japan: Reference values for medical examination. In: Standard program on health checkups and health-maintenance guidance (In Japanese). Ministry of Health, Labour and Welfare of Japan, Tokyo, p59, 2013.

16. Murase N, Katsumura T, Ueda $C$, Inoue $S$ and Shimomitsu T: Validity and reliability of Japanese version of International physical activity questionnaire. J Heal Welf Stat 49: 1-9, 2002 (In Japanese).
17. Craig CL, Marshall AL, Sjöström M, Bauman AE, Booth ML, Ainsworth BE, Pratt M, Ekelund U, Yngve A, Sallis JF and Oja P: International physical activity questionnaire: 12 country reliability and validity. Med Sci Sport Exerc 35: 1381-1395, 2003.

18. Lee PH, Macfarlane DJ, Lam T and Stewart SM: Validity of the international physical activity questionnaire short form (IPAQ-SF): A systematic review. Int J Behav Nutr Phys Act 8: $115,2011$.

19. Hamilton MT, Hamilton DG and Zderic TW: Role of low energy expenditure and sitting in obesity, metabolic syndrome, type 2 diabetes, and cardiovascular disease. Diabetes 56: 2655-2667, 2007.

20. Blair SN, Clark DG and Cureton KJ PK: Exercise and fitness in childhood: Implications for a lifetime health. In: Perspectives in exercise science and sports medicine, Vol 2. Youth exercise and sport. Gisolfi CV and Lamb DL (eds) Benchmark Press, Indianapolis, IN, pp401-430, 1989.

21. Maffeis C, Zaffanello M and Schutz Y: Relationship between physical inactivity and adiposity in prepubertal boys. J Pediatr 131: 288-292, 1997.

22. Yang X, Telama R, Leskinen E, Mansikkaniemi K, Viikari J and Raitakari OT: Testing a model of physical activity and obesity tracking from youth to adulthood: The cardiovascular risk in young Finns study. Int J Obes (Lond) 31: 521-527, 2007.

23. Lantz PM, House JS, Lepkowski JM, Williams DR, Mero RP and Chen J: Socioeconomic factors, health behaviors, and mortality: results from a nationally representative prospective study of US adults. JAMA 279: 1703-1708, 2014.

24. Lahelma E, Martikainen P, Laaksonen M and Aittomäki A: Pathways between socioeconomic determinants of health. J Epidemiol Community Health 58: 327-332, 2004.

25. Nakamura S, Inayama T, Hata K, Matsushita M, Takahashi M, Harada K and Arao T: Association of household income and education with eating behaviors in Japanese adults: A cross-sectional study. BMC Public Health 16: 61, 2016. 